\title{
Brief communication: Possible explanation of the values of Hack's drainage basin, river length scaling exponent
}

\author{
Allen G. Hunt \\ Department of Physics and Department of Earth \& Environmental Sciences, Wright State University, \\ 3640 Colonel Glenn Highway, Dayton, OH 45435, USA \\ Correspondence to: Allen G. Hunt (allen.hunt@wright.edu)
}

Received: 14 May 2015 - Published in Nonlin. Processes Geophys. Discuss.: 18 August 2015

Revised: 22 March 2016 - Accepted: 23 March 2016 - Published: 6 April 2016

\begin{abstract}
Percolation theory can be used to find water flow paths of least resistance. Application of percolation theory to drainage networks allows identification of the range of exponent values that describe the tortuosity of rivers in real river networks, which is then used to generate the observed scaling between drainage basin area and channel length, a relationship known as Hack's law. Such a theoretical basis for Hack's law may allow interpretation of the range of exponent values based on an assessment of the heterogeneity of the substrate.
\end{abstract}

\section{Introduction}

River networks display complex organization as documented in numerous studies. This work addresses only one of them, in particular, the relationship between drainage basin area and river length, which is non-trivial. In Euclidean geometry the basin area, $A$, would be proportional to the square of the river length, $l$; i.e., $l$ should be proportional to $A^{1 / 2}$. In actuality, as determined by Hack (1957), this relationship is

$l=C A^{\beta}$,

with the value of $\beta$ approximately 0.6. Later investigations did not always return the identical value of $\beta$. Nevertheless, Maritan et al. (1996) consider the value of $\beta$ to be well constrained and refer to a study of Gray (1961) as having established that "the accepted values for the exponent $[\beta]$ are in the range 0.57 to 0.6 ".

Hack (1957) (p. 65) asserts that the relationship was a consequence of the lengthening of drainage basins with increasing size. But Montgomery and Dietrich (1992) compare straight-line basin length, $L$, to $A$ over 7 orders of magni- tude of length scale and find precisely $L=A^{0.5}$. That result allows stream length to be expressed in terms of the straightline basin dimension, $l=A^{\beta}=L^{\gamma}=L^{2 \beta}$, so that $\gamma=2 \beta$. The exponent $\gamma>1$ then defines the tortuosity (sometimes known as sinuosity) of the stream path through the drainage basin.

Hack's law explanations have been sought in fractal (Tarboton et al., 1988; Maritan et al., 1996), constructal (Reis, 2006), and "feasible optimality" (Rigon et al., 1998) theories. Fractal theories produce the required self-similar drainage basins (Peckham, 1995) as well as increasing stream sinuosity downstream. I suggest that Hack's law can be understood using percolation theory (Stauffer and Aharony, 1994) because (1) the fractal structure of the percolation cluster generates values for $\gamma$ that constrain the data for $\beta$ reasonably, and (2) it exploits the concept that water flows along paths of minimal resistance, as also in the subsurface (Hunt et al., 2014).

\section{Theory}

There are two distinct applications of percolation theory to flow or conduction problems, and these two applications are those that provide the bounds to Hack's exponent values. The more familiar application is to a binary system, where, e.g., bonds either connect neighboring sites (which in the simplest case are located on a lattice, or grid), or they do not. If enough such neighboring sites are connected, a continuous path of interconnected bonds spans the system. This is denoted the percolation threshold. The shortest distance across the system within this connected cluster is called the chemical path length (Porto et al., 1997). Since all bonds have equal resis- 
tance, the shortest flow path also has the lowest resistance and optimal dissipation.

The second possibility is a system in which bonds of varying resistance connect each pair of neighboring sites. When the system is strongly heterogeneous, i.e., when the distribution of the natural logarithm of the resistances has variance, $\sigma^{2} \gg 1$, the proper application of percolation theory is to find the path of least cumulative resistance. Quantification of this process equates an integral over the local conductance distribution, from a "critical" value, to the largest value, with the percolation threshold (Pollak, 1972). This particular method became known as "critical path analysis", or CPA. The subnetwork so defined is precisely at the percolation threshold (Stauffer and Aharony, 1994). However, the most interesting path across this system is not the shortest, but the optimal path, which provides the least energetic cost in a strongly heterogeneous network (Lopez et al., 2005), i.e., with variance tending to infinity. Since the optimization is not for length, but for energy costs, the path is longer than in a homogeneous system, meaning that its tortuosity exponent is larger. Nevertheless, the chemical path length in a homogeneous system is the analog of the optimal path length in a heterogeneous system, because the shortest path in a system with identical links also represents the path of minimum energy dissipation.

The restriction of river networks to the surface of the earth, and the measurement of stream lengths on 2-D maps, makes the topology of stream connections and the application of percolation theory two-dimensional. In two dimensions, the chemical path length scales with the system size (Sheppard et al., 1999), $L$ as $L^{1.13}$, but the optimal path length scales with system size as $L^{1.21}$, and does not depend on whether a percolation process is classed as random, or invasion (Sheppard et al., 1999). However, not all possible underlying correlations in the local conductance distribution have been investigated. It is known that certain fractal correlation structures in the local conductances can reduce the exponent associated with the conductivity, or reduce the fractal dimensionality of the percolation backbone (Sahimi and Mukhopadhyay, 1996), but there was no corresponding effect noted on the tortuosity or optimal paths exponent. Physical arguments suggest that positive correlations will tend to shorten paths, reducing tortuosity, in accord with the general result that making all conductance magnitudes equal reduces the tortuosity of connected paths. Consequently, one might ask whether negative correlations could lengthen paths. In any case, calling the scaling exponent, $\gamma$, as above, we therefore find that known results from percolation theory constrain its values to be $1.13<\gamma<1.21$. It should be emphasized here that the precision of the numerical calculations of these exponent values by Sheppard et al. (1999) exceeded all other attempts by at least an order of magnitude. Thus it appears that two possible endpoints for the application of percolation theory to the formation of river networks, generated from homogeneous and heterogeneous systems, respectively, constrain observed val- ues of Hack's exponents reasonably well. How could they be realized in nature, or in landscape models?

One can start from an initially homogeneous landscape, and allow stream incision through random headward erosion, analogous to the processes treated in early landscape evolution models (Willgoose et al., 1991), which generate hierarchical structures from random chance associated with rainfall magnitude variability. A connected path with the lowest dissipation (shortest length) will soon acquire the highest flow, through channel erosion feedbacks. Thus, once a river makes a random choice, the enhanced erosion power from the stream reinforces the initial random choice.

The optimal path exponent describes the tortuosity of a channel, when the channel is determined by a global optimization of the flow path in a heterogeneous substrate, and could not be a simple product of headward erosion, which might produce only a local optimization. In such a case geological constraints from varying erodibility can dominate as channels extend either upward, by headward erosion, or downward (e.g., by overtopping of sills).

Using the above result that $\gamma=2 \beta$, we find for Hack's (1957) original result, $l=L^{1.2}$. The range quoted by Maritan et al. (1996), $0.57<\beta<0.6$, generates $1.14<\gamma<1.20$. The predicted range of tortuosity exponent values, $\gamma, 1.13<\gamma<1.21$, generated by percolation theory appears in accord with the observed range of values, and to be slightly larger, consistent with interpreting this range as bounds on observed values.

Note that, while, e.g., Willemin (2000) found a wider range of $\beta$ (0.5 to 0.7$)$ than did Gray (1961), these values were for limited statistics (as small as 11 data points). Although this range is wide, compared with our predictions, when all statistics were put together (Willemin's Fig. 11) the resulting value of $\beta$ was 0.58 . Furthermore, individual values did increase monotonically with increasing geologic heterogeneity. Northwestern Iowa in the middle of the North American craton produced 0.5 , New York, 0.64, and coastal Oregon, in a region of active tectonics, 0.7. Finally, the range of values for $\beta$ quoted by Gray (1961) arose from his consideration of studies over different regions with distinct terrain; uncertainty in a given region was reported in the variation of the numerical prefactor, rather than the exponent.

\section{Conclusions}

Percolation predictions generate a range of exponents consistent with those reported in Hack's law, including the tendency for the largest exponent values to occur in geologically heterogeneous environments.

The statistical nature of percolation theory is in accord with the tendency of the spread in Hack's exponent values to diminish with increasing sample size. 
The source of the tortuosity in the "optimal paths" of lowest energy dissipation is in general accord with the "feasible optimality" (Rigon et al., 1998) proposed to explain Hack's law.

Acknowledgements. I appreciate the dialogue with the editor, Daniel Schertzer.

Edited by: D. Schertzer

Reviewed by: B. Watson and one anonymous referee

\section{References}

Gray, D. M.: Interrelationships of watershed characteristics, J. Geophys. Res., 66 1215-1223, 1961.

Hack, J. T.: Studies of longitudinal profiles in Virginia and Maryland. USGS Professional Papers 294-B, Washington DC, 46-97, 1957.

Hunt, A. G., Ewing, R. P., and Ghanbarian, B.: Percolation Theory for Flow in Porous Media, 3rd Edn., Springer, Berlin, 2014.

Lopez, E., Buldyrev, S. V., Braunstein, L. A., Havlin, S., and Stanley, H. E.: Possible connection between the optimal path and flow in percolation clusters, Phys. Rev. E, 72, 056131, doi:10.1103/PhysRevE.72.056131, 2005.

Maritan, A., Rinaldo, A., Rigon, R., Giacometti, A., and RodriguezIturbe, I.: Scaling laws for river networks, Phys. Rev. E, 53, 1510-1515, 1996.
Montgomery, D. R. and Dietrich, W. E.: Channel initiation and the problem of landscape scale, Science, 255, 826-830, 1992.

Peckham, S. D.: New results for self-similar trees with applications to river networks, Water Resour. Res., 31, 1023-1029, 1995.

Pollak, M.: A percolation treatment of dc hopping conduction, J. Non Cryst. Solids, 11, 1-24, doi:10.1016/0022-3093(72)903043, 1972.

Porto, M., Bunde, A., Havlin, S., and Roman, H. E.: Structural and dynamical properties of the percolation backbone in two and three dimensions, Phys. Rev. E, 56, 1667-1675, 1997.

Reis, A. H.: Constructal view of scaling laws of river basins, Geomorphology, 78, 201-206, 2006.

Rigon, R., Rodriguez-Iturbe, I., and Rinaldo, A.: Feasible optimality implies Hack's law, Water Resour. Res., 34, 3181-3189, 1998.

Sahimi, M. and Mukhopadhyay, S.: Scaling properties of a percolation model with long-range correlations, Phys. Rev. E, 54, 38703880, 1996.

Sheppard, A. P., Knackstedt, M. A., Pinczewski, W. V., and Sahimi, M.: Invasion percolation: new algorithms and universality classes, J. Phys. A: Math. Gen., 32, L521-L529, 1999.

Stauffer, D. and Aharony, A.: Introduction to Percolation Theory, 2nd Edn., Taylor and Francis, London, 1994.

Tarboton, D. G., Bras, R. L., and Rodriguez-Iturbe, I.: The fractal nature of river networks, Water Resour. Res., 24, 1317-1322, 1988.

Willemin, J. H.: Hack's law: Sinuosity, convexity, elongation, Water Resour. Res., 36, 3365-3374, 2000.

Willgoose, G., Bras, R. L., and Rodriguez-Iturbe, I.: A coupled channel network growth and hillslope evolution model. 2 . Nondimensionalization and applications, Water Resour. Res., 27, 1685-1696, 1991. 